\title{
The Vegetation of Isoëto-Nano-Juncetea Class in the Sluch River Valley (Ukraine)
}

\author{
Inna Korotka ${ }^{1 *} \&$ Nataliia Pashkevych ${ }^{2}$
}

${ }^{1}$ M. G. Kholodny Institute of Botany NAS of Ukraine, Tereshchenkivska 2, 01601, Kyiv, Ukraine

${ }^{2}$ Institute for Evolutionary Ecology, NAS of Ukraine, Lebedeva 37, 03143, Kyiv, Ukraine

*corresponding author (e-mail: korotkayainna28@gmail.com)

\begin{abstract}
Vegetation of Isoëto-Nano-Juncetea class has been investigated in the Sluch River valley within the forest-steppe zone. We distinguished three associations belonging to Verbenion supinae (Pulicario vulgaris-Menthetum pulegii Slavnić 1951), Eleocharition ovatae (Cyperetum micheliani Horvatić 1931 and Veronico anagalloidis-Lythretum hyssopifoliae Wagner ex Holzner 1973) alliances. The floristic composition of these syntaxa, is rich in diagnostic species of Bidentetea tripartitae and Phragmito-Magnocaricetea classes, which could indicate a transition to more sustainable communities in the succession row. Our current work is a small portion of a large task to clarify the location of 'hot spots' across the overall diversity of IsoëtoNano-Juncetea, and to identify where and how this diversity can be maintained best.
\end{abstract}

Key words: ephemeral vegetation, phytosociology, Polissia, alien species

\section{Introduction}

Until present, Isoëto-Nano-Juncetea class has remained insufficiently studied in Ukraine as there are only fragmentary researches on this issue (Senchylo \& Honcharenko 2008; Kovalenko 2014). Besides, this class has never been studied on the territory of RightBank Polissia. There are communities of low-growing annual wetland graminoids and some dicot herbs. These species are characterized by a short life cycle and dependence on the hydrological and temperature regimes. Such biological and ecological characteristics enables their survival in the unpredictable conditions of wetlands that can periodically dry out or become flooded. The optimal zone for the communities lies in places where the water level drops down to the bottom level, and then dries out slowly (Chytrý 2011). They are widespread throughout Eurasia, including the Mediterranean region (Šumberová \& Hrivnák 2013; Krawczyk et al. 2016; Mucina et al. 2016). In Ukraine they are commonly found along the banks of the Dnieper River, ponds and other water bodies (Senchylo \& Honcharenko 2008). The research results of floodplain ephemerous vegetation communities in the Pyryatynsky National Nature Park are presented by Kovalenko (2014), in the Horol River and in the planned Korostyshevsky National Nature Park by Orlov \& Yakushenko (2005), and for the whole territory of Ukraine by Solomakha (2008). On the neighboring territories of Russia, research was conducted on the floodplain ephemeretum (Taran 1995), the floodplain of the Ob River (Taran 2001, 2009, 2017) and in the Sudost-Desna River interfluves (Semenyshchenkov 2009). The aim of our study was to classify the dataset of Isoëto-Nano-Juncetea relevés from the Sluch River valley (Ukraine), to make a detailed description of species composition of plant communities.

\section{Material and methods}

We performed an analysis of phytosociological relevés recorded on the territory of the Sluch River valley (Ukraine) (Fig. 1). This dataset included 22 phytosociological relevés of wetland and some types of ruderal vegetation, stored in the phytosociological database format of Turboveg software package (Hennekens \& Schaminée 2001). Most of these relevés (about 70\%) were made on the standard $1-2 \mathrm{~m}^{2}$ plots, or, in several cases, in the areas less than $1 \mathrm{~m}^{2}$. These plots were selected mainly based on the presence of sand banks with 


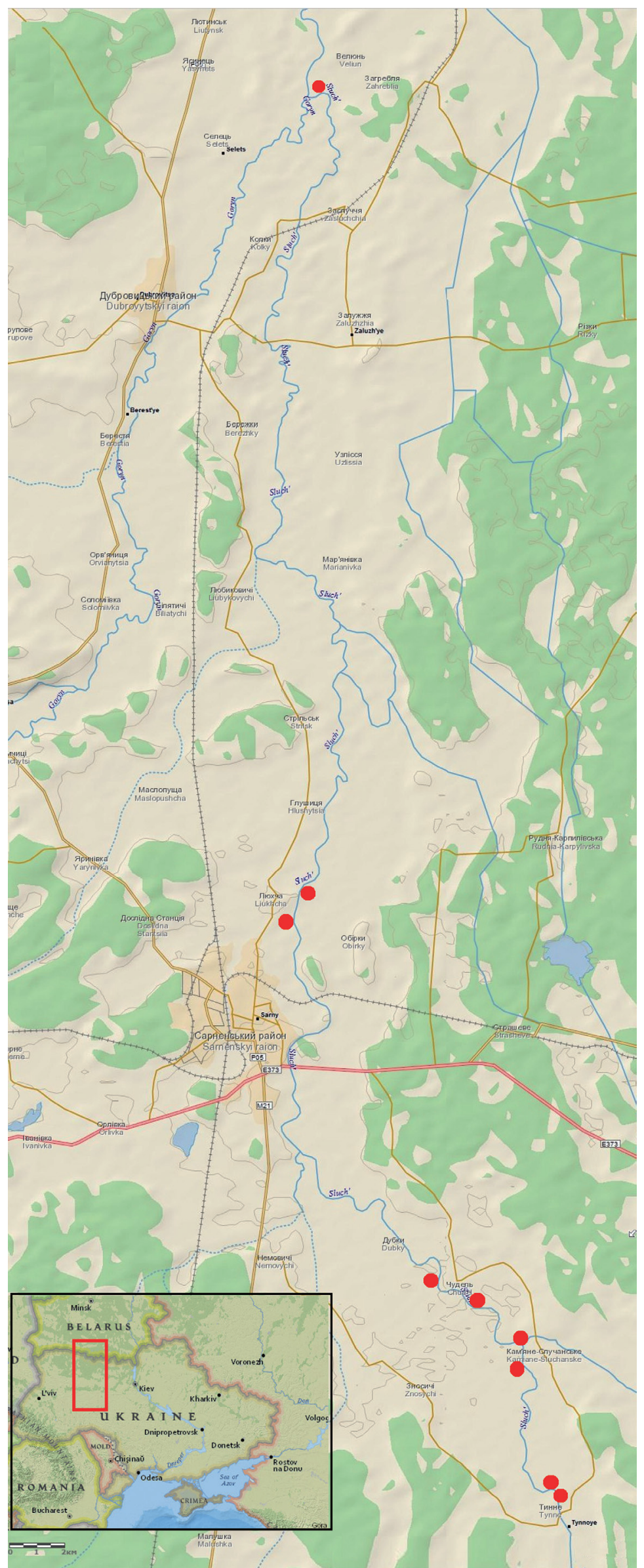

Fig. 1. An overview map of Ukraine with the research area showing the distribution of the vegetation of the Isoëto-Nano-Juncetea class in the Rivne region (Sluch River valley) frequent occurrence of large and homogenous IsoëtoNano-Juncetea stands. The area of research is located downstream the Sluch River in the Rivne region: on the Sarny and Dubrovitsky territories. The river valley is mostly flat and characterized by a broad floodplain, flat banks and alluvial sediments composed of sand, with a constant alluvium flow. These processes lead to the formation of Isoëto-Nano-Juncetea annual communities.

Our research was conducted in August-September 2015, when the riverbed of Sluch is characterized by the lowest water level due to high air temperatures and low rainfall during the summer.

Data processing and classification was carried out using the JUICe software package (Tichý 2002), which was also used for the determination of diagnostic, constant and dominant species. Diagnostic species include differential and characteristic (or indicator) species determined by measuring statistical fidelity using the phi coefficient (Chytrý et al. 2002). Species with phi coefficient above 0.25 were considered diagnostic for a particular association, while species with a phi coefficient above 0.50 were considered highly diagnostic. The latter are underlined in the vegetation description below. Statistical significance of association fidelity was calculated using Fisher's exact test (Chytrý et al. 2002) at a level of significance $\mathrm{P}<0.001$. Constant or highly constant species were those with a frequency over $40 \%$ or $80 \%$, respectively. Dominant and highly dominant species were those with a cover value exceeding $25 \%$ in at least $15 \%$ and $30 \%$ of relevés, respectively. Highly constant and highly dominant species are emphasized in the vegetation description below.

\section{Results}

In the investigated area, the class Isoëto-NanoJuncetea is represented by two alliances and three associations (Table 1).

Isoëto-Nano-Juncetea Br.-Bl. et Tüxen ex Br.-Bl. et al. 1952

Eleocharition ovatae Philippi 1968

Cyperetum micheliani Horvatić 1931

Verbenion supinae Slavnić 1951

Pulicario vulgaris-Menthetum pulegii Slavnić 1951

Veronico anagalloidis-Lythretum hyssopifoliae

Wagner ex Holzner 1973

Vegetation of Isoëto-Nano-Juncetea in the studied area is formed by small therophytes. It develops in late summer and early autumn, in periodically drained areas - on muddy and sandy sediments, silted sand deposits along the rivers, drying bays, oxbow lakes, ponds and lakes (Fig. 2).

The vegetation of the alliance Eleocharition ovatae occurs on riparian sediments along the rivers, oxbow 

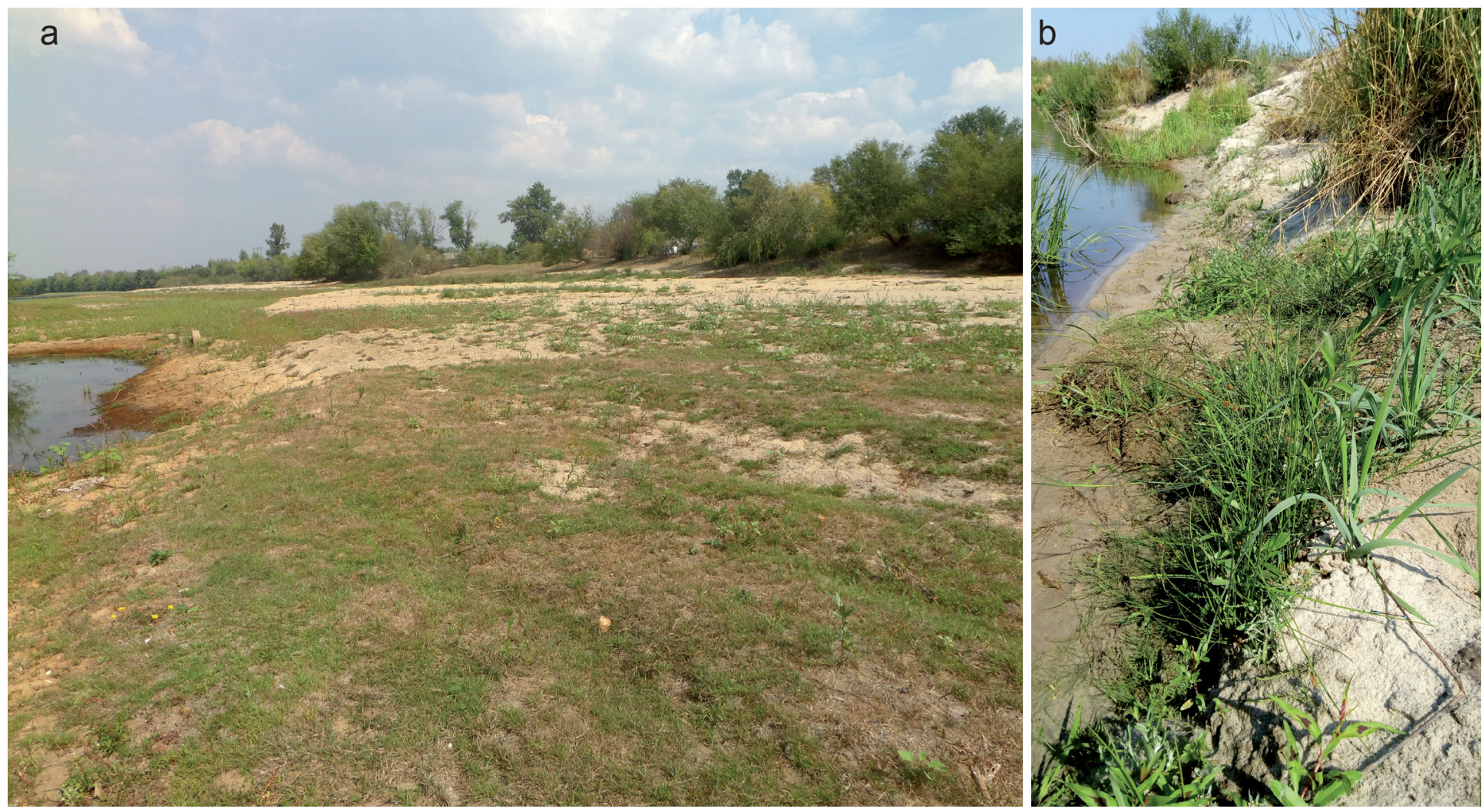

Fig. 2. Sites along the shallow banks of the Sluch River valley with the plant communities of Isoëto-Nano-Juncetea class (Rivne region, Sarny district near Tynne village, right bank of the Sluch River)

Explanations: a - ass. Cyperetum micheliani, b - Veronico anagalloidis-Lythretum hyssopifoliae

lakes and reservoirs on muddy terrain, often with humified substrates composed of gravel or sand. Seed germination requires significant soil moisture or slight flooding, followed by the gradual drying up.

Cyperetum micheliani association includes lowgrowing annual vegetation, formed mainly by Cyperus fuscus L. and Juncus bufonius L. This association includes the low-growing vegetation of summer or winter annuals, or short-lived perennial species, which are weak competitors on sand and muddy substrates. The studied communities occur on sandy riverbanks on flooded, mostly warm lowland areas at the center of the valley, Rivne region. They are rich in the diagnostic species of Bidentetea tripartitae R. Tx., Lohmeyer et Preising 1950 and from the Phragmito-Magnocaricetea Klika in Klika et Novak 1941 class, which probably indicates a transition to more sustainable communities in the succession row.

The alliance Verbenion supinae includes vegetation of low-growing, low-competition annual and perennial species, mainly in disturbed habitats, drained bottoms of ponds, on moist sandy or loamy calcium-rich soils or sandy loams.

The association Pulicario vulgaris-Menthetum pulegii occurs on the outcropping banks of the Sluch River. Patches of this association are found on sandy loam or non-saline soils of the river sediments. Besides the main community edificators, such as: Gnaphalium uliginosum L., and Juncus bufonius L., there are also species characteristic for the eutrophic substrates (Bidens tripartita L., Echinochloa crus-galli (L.) P. Beauv.), and some species of wet meadows (Agrostis stolonifera L.). Pulicario vulgaris-Menthetum pulegii occurs in different habitats, such as: open rivers and ponds, pastures and shallow depressions in alluvial grassland complexes. These habitats are rich in nutrients due to regular flooding or anthropogenic impact (such as agriculture or grazing).

Veronico anagalloidis-Lythretum hyssopifoliae association, represented by low-growing annual vegetation, occurs in small patches in periodically flooded depressions on arable land. This association occurs in narrow strips ( $0.5-10 \mathrm{~m}$ wide) on poor sandy substrates formed by alluvial sediments along the denuded banks of the Sluch River.

In the floristic composition of investigated communities, there are complexes of annual species and the species of Bidentetea tripartitae R. Tx., Lohmeyer et Preising 1950 and Phragmito-Magnocaricetea Klika in Klika et Novak 1941 classes. Eragrostis pilosa (L.) P. Beauv. and Xanthium albinum (Widder) Scholz \& Sukopp are alien species for the studied areas, which 
Table 1. Phytocoenotic table of the Isoëto-Nano-Juncetea class syntaxa and the synoptic table of the Isoëto-Nano-Juncetea plant communities diagnosed within the Sluch River valley. Only species with a frequency $>20 \%$ in the whole dataset and species with a frequency $>40 \%$ in individual associations were presented. Diagnostic species of clusters are shown in bold and ordered by decreasing fidelity, expressed as $\Phi$ values multiplied by 100 (upper index)

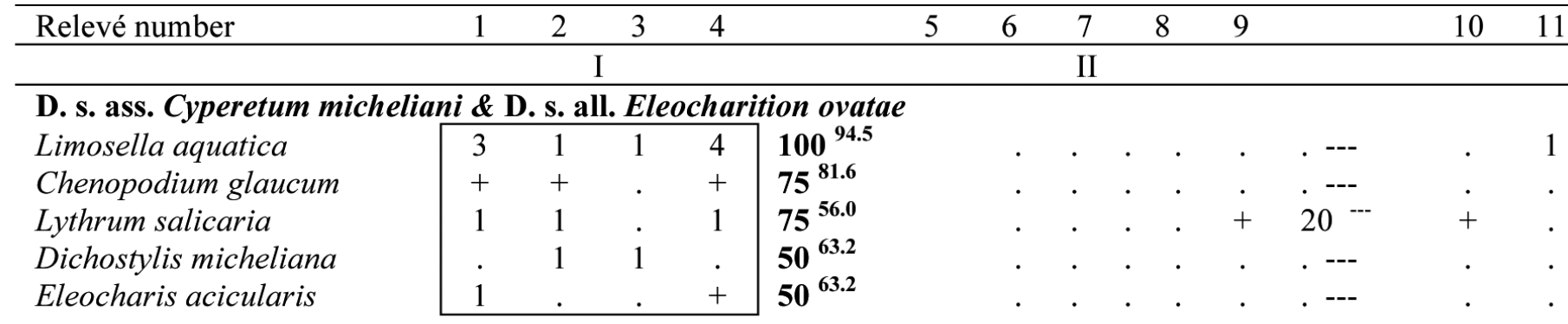

\section{D. s. ass. Pulicario vulgaris-Menthetum pulegii}

Mentha pulegium

Lycopus europaeus

Pulicaria vulgaris

\begin{tabular}{|ccccc|}
\hline 1 & 2 & + & 1 & + \\
+ & 1 &. & 1 & + \\
1 & + &. &. & + \\
\hline
\end{tabular}

10094.5

$80^{63.1}$

$60^{70.7}$

\section{D. s. ass. Veronico anagalloidis-Lythretum hyssopifoliae}

Juncus bufonius

Juncus articulatus

Veronica anagaloides

D. s. all. Verbenion supinae \& D. s. class Isoëto Nano-Juncetea

Gnaphalium uliginosum

Cyperus fuscus

Polygonum persicaria

Other species

Agrostis stolonifera

Eragrostis pilosa

Rorippa amphibia

Plantago major

Xanthium albinum

Echinochloa crusgalli

Bidens frondosa

Bidens tripartita

Salix alba (juv.)

Myosotis palustris

Chenopodium acerifolium

Polygonum hydropiper

Bidens cernua

Erigeron canadensis

Digitaria sanguinalis

Salix acutifolia (juv.)

Herniaria glabra

Juncus tenuis

Chenopodium polyspermum

Potentilla anserina

Leersia oryzoides

Atriplex prostrata

Alisma plantago-aquatica

Bidens radiata

Rumex maritimus

Taraxacum officinale

Oenanthe aquatica

Xanthium strumarium

Ranunculus repens

Inula sabuletorum

Acer negundo

\section{$75^{30.9}$}

\section{- ---}

$1000^{11.5}$

75 --- 3

50

$75^{28.8}$

$75^{26.7}$

$100^{47.7}$

$50^{12.5}$

$50--$

$50^{12.5}$

$75^{58.8}$

$75^{60.8}$

$75^{69.2}$

$75^{60.8}$
. ---

- ---

.---
$60^{16.7}$

$100^{72.5}$

\begin{tabular}{rr}
\hline 1 & 1 \\
1 & 2 \\
+ & 1 \\
\hline
\end{tabular}
3

(

$\begin{array}{llll}1 & 1 & + & 2 \\ 2 & 3 & 1 & 3 \\ 3 & 3 & 4 & 3\end{array}$

$100^{---}$

$100^{11.5}$

$\begin{array}{ll}3 & 1 \\ 1 & 2 \\ 3 & 1\end{array}$

\begin{tabular}{|c|c|c|c|c|c|c|}
\hline 2 & 2 & & 2 & 5 & $80^{16.9}$ & . \\
\hline 2 & & . & $\cdot$ & . & $20^{---}$ & 1 \\
\hline+ & + & . & . & . & $40^{---}$ & + \\
\hline+ & . & . & + & 1 & $60^{---}$ & $\cdot$ \\
\hline & . & $\cdot$ & . & + & $20^{---}$ & . \\
\hline+ & . & . & 1 & + & $60^{14.9}$ & . \\
\hline & . & . & . & 1 & $20^{--}$ & . \\
\hline 1 & 1 & + & . & . & $60^{36.4}$ & 1 \\
\hline . & . & . & . & . &.$^{---}$ & . \\
\hline . & . & + & . & . & $20^{--}$ & . \\
\hline . & . & . & . & . &.$^{--}$ & . \\
\hline+ & . & . & . & . & 20 & . \\
\hline 2 & 3 & . & 2 & + & $80^{50.3}$ & . \\
\hline$\cdot$ & . & . & . & + & $20^{---}$ & . \\
\hline . & . & . & . & + & $20^{---}$ & 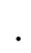 \\
\hline . & . & . & 1 & . & $20^{11.4}$ & 3 \\
\hline 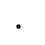 & . & 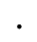 & . & . &.-- & . \\
\hline+ & . & + & + & . & $60^{56.8}$ & 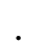 \\
\hline 1 & . & . & . & . & $20^{---}$ & + \\
\hline+ & 1 & 1 & 1 & . & $80^{73.2}$ & . \\
\hline & . & . & . & . & . -- & + \\
\hline 1 & . & + & . & . & $40^{39.3}$ & . \\
\hline . & $\cdot$ & . & . & . & $\dot{0}^{---}$ & + \\
\hline . & 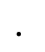 & + & 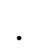 & . & $20^{26.3}$ & . \\
\hline . & + & . & 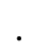 & . & $20^{26.3}$ & $\cdot$ \\
\hline . & + & . & + & 1 & $60^{70.7}$ & . \\
\hline 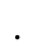 & + & . & 1 & . & $40^{26.0}$ & . \\
\hline 2 & 1 & . & . & . & $40^{55.5}$ & . \\
\hline+ & 1 & $\cdot$ & . & . & $40^{55.5}$ & . \\
\hline 1 & 1 & . & - & . & $40^{55.5}$ & . \\
\hline+ & & & 2 & & $40^{46.6}$ & \\
\hline
\end{tabular}




\begin{tabular}{|c|c|c|c|c|c|c|c|c|c|c|}
\hline 12 & 13 & 14 & 15 & 16 & 17 & 18 & 19 & 20 & 21 & 22 \\
\hline & & & & III & & & & & & \\
\hline
\end{tabular}

\begin{tabular}{lllllllllll|}
\hline 2 & + & 2 & 1 &. & + & + & 3 &. & 2 & + \\
2 & + & 1 & 2 & 1 &. & 1 & 2 & + & 1 &. \\
. & + & 1 &. &. &. & 1 &. &. & 1 &. \\
\hline
\end{tabular}

\begin{tabular}{|c|c|c|c|c|c|c|c|c|c|c|c|}
\hline 2 & 1 & 2 & 2 & 2 & + & 1 & 1 & 2 & 1 & 1 & $100^{--}$ \\
\hline 2 & 2 & 3 & 1 & 1 & 3 & 1 & 3 & & 1 & 1 & $92^{---}$ \\
\hline 1 & 2 & 1 & 4 & 3 & 3 & 1 & . & 1 & + & 1 & $92^{7.3}$ \\
\hline 2 & 4 & 4 & 3 & 3 & 2 & 5 & . & 3 & 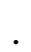 & 2 & $77^{12.2}$ \\
\hline 2 & . & 2 & 3 & 1 & 1 & . & . & 1 & 3 & . & $69^{20.6}$ \\
\hline & 1 & $\cdot$ & . & 2 & . & 1 & . & + & + & . & $54^{--}$ \\
\hline+ & + & . & . & + & . & . & . & + & . & 1 & $46^{---}$ \\
\hline+ & + & . & . & 1 & + & 1 & . & 1 & . & 1 & $54^{18.0}$ \\
\hline & . & . & 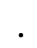 & + & + & 1 & . & + & . & . & $38^{--}$ \\
\hline 1 & + & . & 3 & . & . & 1 & . & 1 & + & 2 & $54^{18.0}$ \\
\hline 1 & 2 & + & . & 2 & . & . & + & . & . & . & $46^{15.9}$ \\
\hline . & . & . & 3 & . & . & . & + & . & + & . & $31^{---}$ \\
\hline . & . & 1 & . & . & . & . & . & . & . & . & $8^{--}$ \\
\hline . & . & . & . & . & . & . & . & . & . & 1 & $15^{--}$ \\
\hline . &. & . & . & . & . & . & . & . & . & 1 & $8^{---}$ \\
\hline . & 1 & . & 1 & 1 & 1 & 2 & . & 1 & . & . & $54^{13.1}$ \\
\hline 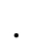 & + & . & 1 & 1 & + & . & . & 1 & + & . & $46^{41.1}$ \\
\hline & + & . & . & 1 & . & . & . & 1 & + & $\cdot$ & $38^{33.9}$ \\
\hline+ & . & . & . & . & $\cdot$ & . & . & . & . & . & $23^{17.6}$ \\
\hline . & . & . & . & 2 & . & + & . & 1 & . & . & $31^{47.8}$ \\
\hline & . & . & . & . & . & . & 2 & . & 2 & . & $15^{---}$ \\
\hline+ & + & + & . & . & . & . & + & . & . & . & $38^{33.9}$ \\
\hline . & $\cdot$ & . & • & + & + & . & . & . & . & . & $15^{--}$ \\
\hline & $\cdot$ & + & . & . & . & . & + & . & . & . & $23^{40.8}$ \\
\hline & + &. & 1 & . &. & $\cdot$ & . & . & . & . & $15^{--}$ \\
\hline & $\cdot$ & . & . & . & . & $\cdot$ & . & $\cdot$ & . & . & $8^{---}$ \\
\hline &. & . & - & . &. &. & + & . & $\cdot$ & . & $8^{---}$ \\
\hline & + & . & . & . & . & $\cdot$ & $\cdot$ & . & . & . & $8^{---}$ \\
\hline &. & . & . & . &. & $\cdot$ & . & . & $\cdot$ & $\cdot$ &.$^{---}$ \\
\hline &. &. & . & . &. & + & . & . & . & . & $8^{---}$ \\
\hline &. & . & $\cdot$ & . &. & . & . & . & $\cdot$ & $\cdot$ &.$^{--}$ \\
\hline & . & . & $\cdot$ & . & . & . & . & . & . & $\cdot$ &.$^{---}$ \\
\hline & $\cdot$ & $\cdot$ & • & · &. & . & $\cdot$ & . & . & . & .--- \\
\hline
\end{tabular}

Sporadic species: Rorippa brachycarpa (2:1), Sedum sexangulare (2:+), Herniaria polygama $(2:+)$, Populus nigra $(2:+)$, Potentilla supina $(2:+)$, Lycopus exaltatus $(1:+, 2:+)$, Myosoton aquaticum (1:+), Crepis tectorum (2:+), Achyrophorus maculatus (1:+), Artemisia marschalliana $(1:+, 2:+)$, Barbarea stricta $(1:+, 2:+)$, Lycopersicon esculentum (1:+, 2:+), Cardamine parviflora (4:+), Typha angustifolia (4:+), Poa annua $(1:+, 2:+)$, Polygonum propinquum (2:+), Galinsoga parviflora (1:+), Juncus atratus (2:+), Mentha arvensis (1:+), Carex acuta (4:+), Sagittaria sagittifolia (4:+), Batrachium trichophyllum (4:+), Spergularia rubra (16:+), Betula obscura (11:+), Lotus corniculatus (11:+), Senecio vernalis (11:+), Trifolium fragiferum (9:1), Trifolium hybridum (9:+), Ranunculus sceleratus (9:+), Erysimum canescens (12:+), Salix purpurea (12:+), Arrhenatherum elatius (12:+), Deschampsia caespitosa (17:+), Leontodon autumnalis (21:+), Lysimachia nummularia (18:+), Lolium perenne (18:+), Artemisia vulgaris (10:+), Epilobium hirsutum $(10:+)$, Cardamine amara $(10:+)$, Scleranthus annuus $(10:+)$, Rorippa palustris $(10:+)$, Artemisia campestre (19:+), Eragrostis minor (19:+), Pimpinella major (15:+), Polygonum bellardii (6:1), Eleocharis palustris (8:1), Elytrigia repens (17:+), Equisetum pratense (17:+), Stenactis annua $(11:+, 1:+)$, Potentilla reptans $(9:+, 13:+)$, Equisetum arvense (13:+,21:+), Galium palustre (20:1), Cerastium arvense $(16:+, 11:+)$, Carex hirta $(8: 1,16: 1)$, Setaria viridis $(17:+, 21: 1)$, Bolboschoenus maritimus (4:+), Eleocharis acicularis (20:+, 21:1), Chenopodium glaucum (20:+,21:+,22:+) Eleocharis ovata (2:+), Salix cinerea $(17: 2,11: 2)$, Rumex confertus (6:+,17:+), Medicago lupulina $(16:+, 12:+, 19:+)$, Polygonum aviculare (16:+,19:+), Plantago lanceolata $(16:+, 15: 1)$, Artemisia absinthium $(16:+, 1:+, 2:+)$, Setaria glauca $(15:+, 21:+)$, Mentha aquatica (6:+,13:1), Portulaca oleracea $(6:+, 1:+, 2:+)$, Gratiola officinalis $(16:+, 11: 1)$, Achillea submillefolium (16:+,15:+,19:+), Inula salicina (16:+,13:2,3:1), Rumex crispus (8:+), Hieracium pilosella $(5:+, 11: 1,10:+)$, Equisetum palustre (20:1,22:1,15:2), Calamagrostis epigeios (19:+,21:+), Cerastium holosteoides $(14:+, 19: 1)$, Veronica anagallis-aquatica (3:2). Juncus conglomeratus $(16: 1,18:+, 21:+)$, Trifolium repens $(9:+, 13:+, 11:+)$, Crypsis alopecuroides (22:1).? Sagina procumbens (16: 1; 20: +), Psammophiliella muralis $(21:+; 10:+)$.

\section{Location of relevés}

Relevé number, Locality, Slope $\left({ }^{\circ}\right)$, Cover total $(\%)$, Latitude $\left({ }^{\circ}\right)$, Longitude $\left(^{\circ}\right)$, Date (year/ month/day), Author abbreviations: NP: Nataliia Pashkevych, IK: Inna Korotka, NA - data not available.

1 - Chudel, Rivne region, Ukraine, $40 \%, 51^{\circ} 15^{\prime} 08.2^{\prime \prime}, \mathrm{N} 26^{\circ} 42^{\prime} 57.0^{\prime \prime} \mathrm{E}$, 22.08.2015, NP

2 - Kaminno-Sluchanske, Rivne region, Ukraine, $20 \%, 51^{\circ} 12^{\prime} 45.6^{\prime \prime}, \mathrm{N}$ $26^{\circ} 45^{\prime} 56.0^{\prime \prime} \mathrm{E}, 22.08 .2015, \mathrm{NP}$

3 - Lyuhcha, Rivne region, Ukraine, $30 \%, 51^{\circ} 22^{\prime} 47.7^{\prime \prime}, \mathrm{N} 26^{\circ} 38^{\prime} 49.5$ "E, 21.08.2015, NP

4 - Lyuhcha, Rivne region, Ukraine, $40 \%, 51^{\circ} 22^{\prime} 47.7^{\prime \prime}, \mathrm{N} 26^{\circ} 38^{\prime} 49.5^{\prime \prime} \mathrm{E}$ 21.08.2015, NP 
5 - Liubykovychy, Rivne region, Ukraine, 60\%, 51 ${ }^{\circ} 29^{\prime} 46.9^{\prime \prime} \mathrm{N} 26^{\circ} 37^{\prime} 34.7^{\prime \prime E}, \mathrm{NP}$

6 - Kolky, Rivne region, Ukraine, 70\%, 51 $35^{\prime} 49.8^{\prime \prime} \mathrm{N} 26^{\circ} 38^{\prime} 22.8^{\prime \prime E}, \mathrm{NP}$

7 - Kolky, Rivne region, Ukraine, 50\%, 51 $35^{\prime} 49.8^{\prime \prime} \mathrm{N} 26^{\circ} 38^{\prime} 22.8^{\prime \prime} \mathrm{E}, \mathrm{NP}$

8 - Kolky, Rivne region, Ukraine, 55\%, 51 ${ }^{\circ} 35^{\prime} 49.8^{\prime \prime} \mathrm{N} 26^{\circ} 38^{\prime} 22.8^{\prime \prime} \mathrm{E}, \mathrm{NP}$

9 - Kaminno-Sluchanske, Rivne region, Ukraine, 60\%, 51 ${ }^{\circ} 12^{\prime} 45.6^{\prime \prime}, \mathrm{N} 26^{\circ} 45^{\prime} 56.0^{\prime \prime} \mathrm{E}, 22.08 .2015$, NP

10 - Lyuhcha, Rivne region, Ukraine, 60\%, 51²2'19.8", N 26³8'20.7"E, 21.08.2015, IK

11 - Tynne, Rivne region, Ukraine, 50\%, 51 ${ }^{\circ} 10^{\prime} 25.4^{\prime}, \mathrm{N}^{\circ} 6^{\circ} 46^{\circ} 41.0^{\prime \prime} \mathrm{E}, 22.08 .2015$, NP

12 - Lyuhcha, Rivne region, Ukraine, 60\%, 51 ${ }^{\circ} 23^{\prime} 03.7^{\prime \prime}, \mathrm{N} 26^{\circ} 39^{\prime} 06.0^{\prime \prime} \mathrm{E}, 21.08 .2015$, IK

13 - Kaminno-Sluchanske, Rivne region, Ukraine, $5^{\circ}$ SSW, 60\%, 51 ${ }^{\circ} 12^{\prime} 55.9^{\prime \prime}$, N 264 $45^{\prime} 57.1^{\prime \prime} \mathrm{E}, 22.08 .2015$, NP

14 - Wielun, Rivne region, Ukraine, 65\%, 51³7'59.4", N 26³9'17.4"E, 21.08.2015, IK

15 - Chudel, Rivne region, Ukraine, 60\%, 51 ${ }^{\circ} 15^{\prime} 08.2^{\prime \prime}, \mathrm{N} 26^{\circ} 42^{\prime} 57.0^{\prime \prime} \mathrm{E}, 22.08 .2015$, NP

16 - Tynne, Rivne region, Ukraine, 35\%, 51 ${ }^{\circ} 10^{\prime} 24.9^{\prime \prime} \mathrm{N}, 26^{\circ} 46^{\prime} 41.3 " \mathrm{E}, 22.08 .2015$, NP

17 - Lyuhcha, Rivne region, Ukraine, 40\%, 51 ${ }^{\circ} 22^{\prime} 47.7^{\prime \prime}, \mathrm{N} 26^{\circ} 38^{\prime} 49.5^{\prime \prime} \mathrm{E}, 21.08 .2015$, NP

18 - Tynne, Rivne region, Ukraine, 80\%, 51 10'23.9", N 26046'39.9"E, 22.08.2015, IK

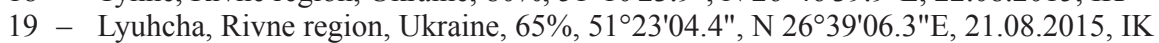

20 - Tynne, Rivne region, Ukraine, 40\%, 51 ${ }^{\circ} 10^{\prime} 25.4^{\prime \prime}, \mathrm{N} 26^{\circ} 46^{\prime} 41.0^{\prime \prime E}, 22.08 .2015$, NP

21 - Wielun, Rivne region, Ukraine, 30\%, 51³7'59.4", N 26³9'17.4"E, 21.08.2015, IK

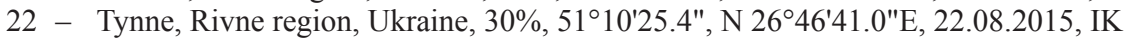

have recently spread and naturalized in different phytocoenoses, including the communities of Isoëto-NanoJuncetea class.

\section{Discussion}

Analysis of data on the Isoëto-Nano-Juncetea class communities currently available in Ukrainian (Senchylo \& Honcharenko, 2008) and other literature sources (Holub et al. 2007; Kovalenko 2014) showed that the series of relevés done by us in the Sluch River valley differs from already existing syntaxa associations, both in floristic composition and in habitat conditions (Table 2). Also, for the comparison of associations, we have also used literature sources dealing with studies outside the territory of research and adjacent territories (Brullo \& Minissale 1998; Popiela et al. 2009).

The aquatic environment is a corridor for the transfer of seeds, including those of alien species. Therefore, it is necessary to pay more attention to the assessment of the alien species influence on vegetation formation, especially, on ephemeral vegetation.

In the floristic composition of the investigated communities, many of such species, belonging to different families, were recorded. However, the most common are neophytes characterized by the $\mathrm{C}_{4}$ carbon fixation. As known, the total number of $\mathrm{C}_{4}$ plants represents about 3\% of all land plants (Simpson 2010) and the majority of these plant species in Europe (Pyankov et al. 2010) belong to four families: Amaranthaceae, Chenopodiaceae, Cyperaceae and Poaceae. The analysis of systematic structure of the studied vegetation class has shown this trend as well. Among the species of Isoëto-Nano-Juncetea class characterized by the $\mathrm{C}_{4}$ carbon fixation are: Atriplex prostrata R. Br., Chenopodium acerifolium Andrz., Ch. polyspermum L., (Chenopodiaceae), Agrostis stolonifera L., Digitaria sanguinalis (L.)
Scop., Echinochloa crus-galli (L.) Scop., Eragrostis minor Host, E. pilosa (L.) P. Beauv., Leersia oryzoides (L.) Sw., Setaria glauca (L.) P. Beauv., S. viridis (L.) P. Beauv. (Poaceae) etc. A favorable factor for the invasion of these species into the community of ephemeretum may be an increase in drought and environmental degradation in the study region.

In the Sudost-Desnyanskiy interfluves, Semenyschenkov (2009) observed one association belonging to the class (Cypero fusci-Limoselletum) that includes three communities, one of which is Juncus bufonius community.

On the flood plain of the River Ob, Taran (2017) reported the presence of annual shoots of Salix alba and S. triandra in the Cypero fusci-Limoselletum association. In the plant communities occurring on the River Sluch banks, the juvenile individuals of willow species of Salix alba and S. acutifolia have been discovered.

Šumberová \& Hrivnak (2013) has pointed out that the Isoëto-Nano-Juncetea class represents a very interesting mix of different communities, 'which include dozens of associations described from different parts of Europe and the transitions between them across relatively small regions of Europe'. Our work is only a small portion of an in-depth investigation to clarify the location of 'hot spots' across the whole diversity of Isoëto-Nano-Juncetea, and to understand where and how this diversity can be best maintained.

The flood-plain ephemeretum of the River Sluch is represented by three associations of two alliances of the Nanocyperetalia Klika 1935 order, Isoëto-NanoJuncetea Br.-B1. et Tx. in Br.-B1. et al. 1952 class. Thus, for the class of ephemeral vegetation, there is a significant threat of the alien species introduction, which over time and during structural transformations can be on a significant scale and even result in new syntaxa formation. In the shallows, Cyperetum micheliani ass. 
Table 2. Synoptic table of the Isoëto-Nano-Juncetea plant communities diagnosed in Ukraine. Only species with a frequency $>10 \%$ in the whole dataset and species with a frequency $>20 \%$ in individual associations were presented. Diagnostic species of clusters are in bold and ordered by decreasing fidelity, expressed as $\Phi$ values multiplied by 100 (upper index). The relevés of plant communities in this table were made by O. Kovalenko (2014) (Group No. 1-5) and the authors (Group No. 6-8)

\begin{tabular}{|c|c|c|c|c|c|c|c|c|}
\hline Group No. & 1 & 2 & 3 & 4 & 5 & 6 & 7 & 8 \\
\hline No. of relevé & 8 & 5 & 8 & 12 & 11 & 7 & 12 & 5 \\
\hline Eragrostis suaveolens & $100^{100.0}$ &.-- &.$^{---}$ &.$^{---}$ &.-- &.-- &.$^{---}$ &.$^{---}$ \\
\hline Crypsis alopecuroides & $100^{95.5}$ &.-- &.-- &.-- &.-- &.-- & $8^{--}$ &.-- \\
\hline Convolvulus cantabrica & $8^{92.7}$ & -- & --- & --- & -- & --- &.-- & -- \\
\hline Polygonum aviculare & $62^{57.5}$ & $20^{8.7}$ & ${ }^{---}$ &.-- &.-- & ${ }^{---}$ & $17^{4.9}$ & $\cdot--$ \\
\hline Spergularia rubra & $38^{27.1}$ & $60^{52.2}$ & -- & --- & --- & --- & $8^{---}$ & --- \\
\hline Psammophiliella muralis & $100^{59.7}$ & $100^{59.7}$ & $12^{--}$ & $\cdot--$ &.-- & ${ }^{---}$ & $17^{---}$ & ${ }^{---}$ \\
\hline Radiola linoides & -- & $80^{88.2}$ &.-- & --- & --- & --- & $\cdot--$ & -- \\
\hline Veronica scutellata & ${ }^{---}$ & $60^{75.3}$ & -- & -- & -- & -- & -- & -- \\
\hline Myosurus minimus & -- & $80^{88.2}$ & -- & --- & -- &.-- & --- & --- \\
\hline Gnaphalium rossicum & $38^{15.8}$ &.-- & $100^{74.2}$ & --- & $7^{6.2}$ & -- & -- & -- \\
\hline Lythrum hyssopifolia & $12^{4.1}$ & -- & $62^{68.9}$ & --- & -- & -- & --- & -- \\
\hline Elatine alsinastrum &.-- & $-\cdots$ & $50^{58.3}$ & --- &.-- & $14^{8.7}$ & --- & -- \\
\hline Rumex maritimus &.-- & -- & $25^{27.8}$ & --- & -- &.-- & $8^{2.5}$ & $20^{20.2}$ \\
\hline Potentilla supina &.-- &.-- & $62^{60.8}$ & --- & -- & $29^{20.5}$ &.-- & -- \\
\hline Bolboschoenus maritimus & -- & -- & $38^{26.6}$ & $42^{31.2}$ & -- & $29^{16.7}$ & -- & -- \\
\hline Dichostylis micheliana & -- & -- & $75^{64.3}$ &.$^{--}$ & -- & $43^{30.0}$ & --- & --- \\
\hline Pycreus flavescens & -- & -- &.$^{---}$ & $100^{100.0}$ & $-\cdots$ &.-- & $-\cdots$ & -- \\
\hline Juncus gerardii & -- & $60^{50.8}$ & --- & $\mathbf{5 0}^{39.8}$ & -- &.- & $\cdot--$ & -- \\
\hline Carex distans & $-\cdots$ &.-- & --- & $75^{85.1}$ & --- & $-\cdots$ &.-- & --- \\
\hline Trifolium fragiferum & -- & -- & -- & $92^{84.8}$ & -- & -- & --- & $20^{6.6}$ \\
\hline Schoenoplectus elanospermus &.-- &.-- & $25^{9.4}$ & $75^{61.1}$ & $27^{11.7}$ & --- & --- &.-- \\
\hline Puccinelia distans & -- & -- &.- & $50^{68.3}$ &.-- & -- & -- & -- \\
\hline Sagina nodosa & $-\cdots$ & $-\cdots$ & --- & $58^{54.4}$ & $36^{28.7}$ & $-\cdots$ & $-\cdots$ & --- \\
\hline Juncus bufonius & $75^{19.7}$ & $40^{--}$ & $25-$ & $42--$ & $100^{38.6}$ & $43-$ & $67^{13.4}$ & -- \\
\hline Sagina procumbens &.$^{---}$ & $20--$ & -- & $83^{52.4}$ & $73^{43.0}$ & $\cdot--$ & $17^{---}$ & -- \\
\hline Juncus tenuis & -- &.-- & -- & $42^{18.4}$ & $55^{30.2}$ & $-\cdots$ & $17^{--}$ & $60^{35.3}$ \\
\hline Juncus compressus &.-- & ${ }^{---}$ & -- &.-- & $36^{57.7}$ & -- & $\cdot-$ &.-- \\
\hline Limosella aquatica & $-\cdots$ & $\cdots$ & $50^{22.5}$ & -- & $45^{18.5}$ & $100^{66.5}$ & --- & -- \\
\hline Lythrum salicaria &.- & ${ }^{---}$ &.$^{--}$ & -- &.-- & $43^{41.6}$ & $7^{8.5}$ & $20^{12.7}$ \\
\hline Chenopodium glaucum & $-\cdots$ & $-\cdots$ & --- & -- & -- & $43^{62.9}$ & 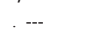 &.-- \\
\hline Myosotis palustris & -- & -- & -- & -- & -- & $57^{56.8}$ & $8^{--}$ & $20^{11.4}$ \\
\hline Eleocharis acicularis & -- & -- & $38^{25.6}$ & $8--$ & $9-$ & $57^{47.0}$ &.-- & . --- \\
\hline Chenopodium acerifolium &.- & -- & $38^{24.4}$ &.-- & -- & $71^{60.7}$ & $8^{---}$ & --- \\
\hline Juncus articulatus & -- & -- & ${ }^{---}$ & -- & $-\cdots$ & $14^{---}$ & $83^{60.5}$ & $60^{38.3}$ \\
\hline Digitaria sanguinalis & -- & $\cdots$ & -- & -- & -- & $-\cdots$ & $33^{40.4}$ & $20^{20.2}$ \\
\hline Chenopodium polyspermum & -- & -- & -- & -- & -- & -- & $42^{48.1}$ & $20^{17.4}$ \\
\hline Veronica anagaloides & $-\cdots$ & $-\cdots$ & -- & -- & -- & $-\cdots$ & $42^{23.7}$ & $100^{81.5}$ \\
\hline Lycopus europaeus & $-\cdots$ & $\cdots$ & $12--$ & -- & $\cdots$ & $-\cdots$ & $33^{18.3}$ & $80^{66.7}$ \\
\hline Pulicaria vulgaris & $12^{--}$ & -- & $25^{13.0}$ & -- & $9^{---}$ & -- &.-- & $60^{51.9}$ \\
\hline Potentilla anserina & --- & $40^{21.5}$ &.-- & $8--$ & -- & $-\cdots$ & $17^{--}$ & $80^{60.7}$ \\
\hline Mentha pulegium & -- &.-- & -- &.-- & -- & $-\cdots$ & $8--$ & $100^{95.5}$ \\
\hline Eragrostis pilosa &.-- & -- & --- & --- & --- & $43^{27.4}$ & $67^{51.8}$ & $20^{3.9}$ \\
\hline Bidens cernua & -- & -- & -- & $8--$ & -- &.-- & $50^{32.7}$ & $80^{62.7}$ \\
\hline Rorippa amphibia & -- & $-\cdots$ & -- &.-- & -- & $43^{26.7}$ & $50^{33.9}$ & $40^{23.8}$ \\
\hline Polygonum persicaria & -- & $\cdots$ & -- & -- & $18^{---}$ & $43^{9.2}$ & $2^{48.8}$ & $100^{55.6}$ \\
\hline Gnaphalium uliginosum & -- & -- & --- & --- &.-- & $71^{29.9}$ & $10^{52.7}$ & $100^{52.7}$ \\
\hline Agrostis stolonifera & -- & $-\cdots$ & -- & $17^{--}$ & -- & $29^{3.1}$ & $75^{43.6}$ & $80^{48.0}$ \\
\hline Bidens frondosa & $-\cdots$ & $60^{27.4}$ & $25--$ &.-- & -- & $57^{25.0}$ & $58^{6.0}$ & $20--$ \\
\hline Cyperus fuscus & $38-$ &.-- & $88^{20.2}$ & $75^{10.5}$ & -- & $100^{29.9}$ & $92^{23.5}$ & $100^{29.9}$ \\
\hline Plantago major & -- & $80^{14.9}$ & $100^{30.3}$ & $33^{---}$ & $100^{30.3}$ & $71^{8.2}$ & $42--$ & $60^{---}$ \\
\hline Centaurium pulchellum & $\cdots$ & $100^{51.9}$ & $75^{32.0}$ & $75^{32.0}$ & $27^{---}$ &.-- &.-- &.-- \\
\hline Chenopodium rubrum & $12^{--}$ & $-\cdots$ & $38^{28.6}$ & $50^{42.9}$ & $-\cdots$ & -- & -- & -- \\
\hline Atriplex prostrata &.-- & -- & --- &.$^{---}$ & -- & $\cdots$ & $17^{9.1}$ & $60^{64.7}$ \\
\hline Erigeron canadensis & -- & -- & -- & -- & -- & -- & $50^{55.2}$ & $20^{15.0}$ \\
\hline Echinochloa crusgalli & $12-$ & $\cdots$ & --- & -- & -- & $29^{11.9}$ & $33^{16.7}$ & $60^{43.7}$ \\
\hline Xanthium albinum & --- & -- & -- & -- & -- & $43^{29.2}$ & $58^{45.5}$ & $20^{5.1}$ \\
\hline Bidens tripartita & -- & $\cdots$ & -- & -- & $36^{17.7}$ &.-- & $50^{31.0}$ & $60^{40.8}$ \\
\hline Polygonum hydropiper & $\cdots$ & $\cdots$ & $25^{11.7}$ & $8^{---}$ & $9^{---}$ & $43^{31.0}$ & $8^{---}$ & $20^{6.3}$ \\
\hline Alisma plantago-aquatica & $\cdot-$ & $\cdot-$ &.- &.-- & $9^{5.4}$ & $29^{37.1}$ & $8^{4.2}$ & -- \\
\hline Inula salicina & -- & -- & -- & -- & --- & $29^{37.5}$ & $17^{18.0}$ & -- \\
\hline Oenanthe aquatica & -- & $\cdots$ & -- & $17^{8.5}$ & -- & $14^{5.5}$ & $8^{---}$ & $40^{38.1}$ \\
\hline Polygonum scabrum & $50^{68.3}$ & $-\cdots$ & -- &.-- & -- &.-- &.-- & \\
\hline
\end{tabular}

Explanations: 1 - Eragrostidetum suaveolentis, 2 - Psammophilello-Juncetum nastant, 3 - Eleochario acicularis-Limiselletum aquaticae, 4 - Cyperetum flavescentis, 5 - Juncetum bufonii, 6 - Cyperetum micheliani, 7 - Veronico anagalloidis-Lythretum hyssopifoliae, 8 - Pulicario vulgaris-Menthetum pulegii 
dominates, while the associations of Verbenion supinae Slavnić 1951 alliance often dominate in disturbed habitats, such as, wet places in pastures and on the river banks.

Acknowledgments. We express our gratitude to Dr. Andriy Mosyakin for assisting in editing of the translation,
Dr. Yuliia Mala for assisting in field material collection, which greatly improved the manuscript. We also thank anonymous reviewers for their helpful comments and suggestions, which significantly improved the quality of this research paper.

\section{References}

Brullo S. \& Minissale P. 1998. Considerazioni sintassonomiche sulla classe Isoëto-Nanojuncetea. Itinera Geobotanica 11: 263-290.

Chytrý M. 2011. Vegetation of the Czech Republic. Aquatic and wetland vegetation. Vyd. 1: 828 .

Chytrý M., Tichý L., Holt J. \& Botta-Dukát Z. 2002. Determination of diagnostic species with statistical fidelity measures. J Veg Sci 13: 79-90. https://doi. org/10.1111/j.1654-1103.2002.tb02025.x

Hennekens S. M. \& Schaminée J. H. J. 2001. TURBOVEG, a comprehensive data base management system for vegetation data. J Veg Sci. 12(4): 589-591. http:// dx.doi.org/10.2307/3237010

Holub V. B., Dubyna D. V. \& Kuz'myna E. V. 2007. Communities of Eragrostidetum suaveolentis ass. nova in the valley of the Lower Volga. Samara Bend. 16 (3) (21): 532-537.

Kovalenko O. 2014. Syntaxonomy of flood-plain ephemerous vegetation (Isoëto-Nano-Juncetea of the National Nature Park "Pyryatynsky" (Poltava region, Ukraine). Botanical Journal 99(1): 34-60.

Krawczyk R., Cwener A., Michalczuk W. \& Zubel R. 2016. Ephemeral wetland communities of IsoëtoNano-Juncetea class - new data from south-eastern Poland. Biodiv. Res. Conserv. 42: 41-54. https://doi. org/10.1515/biorc-2016-0007

Mucina L., Bültmann H., Dierssen K., Theurillat J.-P., Raus T., Čarni A., Šumberová K., Willner W., Dengler J., Gavilán García R., Chytrý M., Hájek M., Di Pietro R., Iakushenko D., Pallas J., Daniëls F. J. A., Bergmeier F. E., Santos Guerra A., Ermakov N., Valachovič M., Schaminée J. H. J., Lysenko T., Didukh Ya. P., Pignatti S., Rodwell J. S., Capelo J., Weber H. E., Solomeshch A., Dimopoulos P., Aguiar C., Hennekens S. M., TichÝ L. 2016. Vegetation of Europe: hierarchical floristic classification system of vascular plant, bryophyte, lichen, and algal communities. App Veg Sci, 19(Suppl. 1): 3-264.

Orlov O. O. \& Yakushenko D. M. 2005. Vegetation of the projected Korostyshevsky National Park. 180 pp.

Popiela A., Prajs B. \& Łysko A. 2009. New data on the distribution of dwarf ephemeral wetland vascular plant species and communities in western and northwestern Poland. Biodiv. Res. Conserv. 15: 41-46. DOI: 10.2478/v10119-009-0016-0

Pyankov V.I., Ziegler H., Akhani H., Deigele C. \& Lüttge U. 2010. European plants with $\mathrm{C}^{4}$ photosynthesis: geographical and taxonomic distribution and relations to climate parameters. Bot J Linn Soc 163(3): 283-304.

Semenyshchenkov Yu. A. 2009. Phytocenotic diversity of Sudost-Desnyanskogo interfluves, pp. 68-70.

Senchylo O. O. \& Honcharenko I. V. 2008. Isoëto-NanoJuncetea of the limitation bare of the steppe zone Dnipro. Bulletin of Donetsk National University, Series A: Natural sciences 2: 334-343.

Simpson M. G. 2010. Plant Systematics, 2nd Edition. 752 pp. Academic Press.

SolomakHA V. A. 2008. Syntaksonomiia roslynnosti Ukrajiny. Tretie nablyzhennia. 296 pp. Fitosociocentr, Kyiv.

ŠumberovÁ K. \& HrivnÁK R. 2013. Formalised classification of the annual herb vegetation of wetlands (IsoëtoNano-Juncetea class) in the Czech Republic and Slovakia (Central Europe). Phytocoenologia 43 (1-2): 13-40. DOI: 10.1127/0340-269X/2013/0043-0529

TARAN H. S. 1995. A little known vegetation class of the former USSR - flood-plain Ephemeretum (IsoëtoNanojuncetea Br.-Bl. et Tx. 43). Siberian Journal of Ecology 2(4): 372-380.

TARAN H. S. 2001. Association Cypero-Limoselletum (Oberd. 1957) Korneck 1960 (Isoëto-Nano-Juncetea) in the floodplain of middle $\mathrm{Ob}$. Vegetation of Russia 1: 43-56.

TARAN H. S. 2009. Bottomland ephemeretum of $\mathrm{Ob}$ and Irtysh Rivers near Khanty-Mansiysk city. Vestnik of the Orenburg State University. 2: 108-110.

TARAN H. S. 2017. Ephemeral Wetland Vegetation of the $\mathrm{Ob}$ River in the Forest-Steppe Zone of Western Siberia. Journal of Siberian Federal University. Biology, p. 1-17. DOI: $10.17516 / 1997-1389-0032$

TICHÝ L. 2002. JUICE, software for vegetation classification. J Veg Sci 13: 451-453. 